\title{
Upregulation of microRNA-138-5p inhibits pancreatic cancer cell migration and increases chemotherapy sensitivity
}

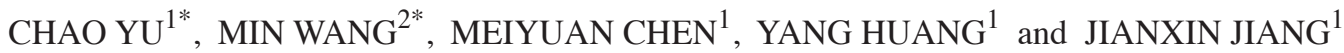 \\ ${ }^{1}$ Department of Hepatobiliary Surgery, Affiliated Hospital of Guiyang Medical College, Guiyang, Guizhou 550004; \\ ${ }^{2}$ Department of Biliary-Pancreatic Surgery, Affiliated Tongji Hospital, Tongji Medical College, \\ Huazhong University of Science and Technology, Wuhan, Hubei 430000, P.R. China
}

Received July 19, 2014; Accepted March 26, 2015

DOI: $10.3892 / \mathrm{mmr} .2015 .4031$

\begin{abstract}
The present study investigated the role of microRNA (miR)-138-5p in regulating carcinoma migration and sensitivity to chemotherapy in pancreatic cancer. Reverse transcription-quantitative polymerase chain reaction (RT-qPCR) was used to assess the expression levels of miR-138-5p in pancreatic cancer cell lines and primary carcinoma tissues from human patients. A lentiviral vector, containing miR-138-5p mimics (lv-miR-138-m) or miR-138-5p inhibitor (lv-miR-138-i), was used to either upregulate or downregulate the expression levels of miR-138-5p in PANC-1 cells, respectively. The effects of miR-138-3p regulation on pancreatic cancer cell migration and sensitivity to chemotherapy were examined. The predicted targeting of miR-138-5p on vimentin (VIM) was assessed by western blotting in PANC-1 cells. VIM was subsequently downregulated using small interfering (si)RNA to determine its effect on miR-138-5p-modulated pancreatic cancer cell development. The expression levels of miR-138-5p were downregulated in pancreatic cancer cell lines and primary carcinoma tissues. In PANC-1 cells, lentivirus-mediated upregulation of miR-138-5p inhibited cancer cell migration and increased cell chemosensitivity to 5-fluorouracil (5-FU). By contrast, downregulation of miR-138-5p promoted cancer cell migration and decreased cell chemosensitivity to 5-FU. A luciferase assay revealed that VIM was a direct target of miR-138-5p. Western blotting demonstrated that VIM was downregulated upon the upregulation of miR-138-5p in PANC-1 cells. siRNA-mediated downregulation of VIM inhibited pancreatic cancer cell migration in the control and miR-138-5p downregulated PANC-1 cells. The present study
\end{abstract}

Correspondence to: Dr Jianxin Jiang, Department of Hepatobiliary Surgery, Affiliated Hospital of Guiyang Medical College, 28 Guiyi Street, Guiyang, Guizhou 550004, P.R. China

E-mail: jjx731@aol.com

*Contributed equally

Key words: microRNA-138-5p, vimentin, pancreatic cancer, MTT, western blotting demonstrated that miR-138-5p is important in regulating pancreatic cancer development, possibly through targeting VIM.

\section{Introduction}

Pancreatic cancer is one of the most deadly forms of carcinoma, estimated to be the seventh leading cause of cancer-related mortality in China and the fourth leading cause of cancer-related mortality in the United States $(1,2)$. In the United States alone, $>250,000$ patients succumb to pancreatic cancer annually (3). Although progress has been made in understanding the molecular mechanisms and developing suitable treatment strategies, the overall prognosis of patients with pancreatic cancer remains poor $(4,5)$. Therefore, it is of great interest to further understand the development of pancreatic cancer and to seek novel methods for early detection and effective therapeutic strategies to treat patients with pancreatic cancer.

MicroRNAs (miRNAs) are a group of non-coding, small-size RNA nucleotides involved in regulating gene expression through translational cleavage or repression (6). In previous decades, it has been demonstrated that miRNAs are widely involved in various developmental stages, including embryogenesis, organ maturation, cell differentiation and carcinoma, in human and animal tissue (7-9). In cancer, mounting evidence has revealed that miRNAs are directly involved in several aspects of cancer development, including carcinogenesis, tumor growth, tumor differentiation, carcinoma metastasis and cancer apoptosis (7,10-15).

Among several of the cancer-associated miRNAs (miR), miR-138 is a family of microRNA precursors. The overexpression or underexpression of miR-138 have been demonstrated to be important in regulating tumor development or cancer apoptosis in various forms of carcinoma, including lung cancer (16), hepatocellular carcinoma (17) and leukemia (18). However, little is known regarding the expression pattern or functional role of miR-138 in modulating pancreatic cancer development.

In the present study, the expression levels of the human isoform of miR-138, miR-138-5p, were examined in human pancreatic cancer cell lines and primary pancreatic carcinoma, obtained from patient samples. The possible biological 
functions of miR-138-5p in regulating cell invasion or sensitivity to the chemotherapeutic agent, 5-fluorouracil (5-FU), were investigated through lentivirus-mediated miR-138-5p over- or downregulation in the PANC-1 pancreatic cancer cell line. The possible molecular target, vimentin (VIM), was examined to determine whether it was directly regulated by miR-138-5p in pancreatic cancer. Small interfering (si) RNA-mediated genetic downregulation of VIM was also performed to observe whether VIM exerted any functional role in regulating pancreatic cancer development.

\section{Materials and methods}

Cell lines and culture. A total of eight pancreatic cancer cell lines were used: AsPC-1, BxPc-3, Capan-1, Capan-2, CFPAC-1, PANC-1, MIA PaCa-2 and SW1990 (American Type Culture Collection, Shanghai, China). Human pancreatic ductal epithelial (HPDE) cells were obtained from Dr M.S. Tsao at the Ontario Cancer Institute (Ontario, Canada) and maintained as described previously (19). Primary human normal pancreatic epithelial cells were obtained from Shanghai Genomics (Shanghai, China) and cultured in CS-C medium (Cell Systems Corporation, Kirkland, WA, USA), containing $10 \%$ fetal bovine serum (FBS; Sigma-Aldrich, St. Louis, MO, USA).

Tissue samples. The human pancreatic tissue samples were obtained from patients following a surgical procedure to resect a portion of the pancreas, performed at the Department of Hepatobiliary Surgery, Affiliated Hospital of Guiyang Medical College (Guiyang, China) between January 2013 and March 2014. The normal pancreatic tissue samples were obtained from areas of peripheral tissue adjacent to tumors. The tissues were rapidly removed and a section of each sample was embedded in Optimal Cutting Temperature compound (Miles Laboratories, Elkhart, IN, USA). Histologic examination was subsequently performed on all tissue samples adjacent to the specimens (BX51WI; Olympus Corporation, Tokyo, Japan). Consent forms were obtained from all patients and all procedures in the present study were reviewed and approved by the Ethics Committees of the Affiliated Hospital of Guiyang Medical College and Affiliated Tongji Hospital.

Lentivirus construction and transfection. The oligonucleotides of the hsa-miR-138-5p mimics, hsa-miR-138-5p inhibitor, and their non-specific control were synthesized by RiboBio (RiboBio, Shanghai, China). The coding sequences were subsequently amplified and cloned into pCDH-CMV-MCS-EF1-coGFP constructs (System Biosciences, Mountain View, CA, USA). The lentiviral expression constructs and pPACK packaging plasmid mix (System Biosciences) were co-transfected into $293 \mathrm{~T}$ cells (American Type Culture Collection), according to the manufacturer's instructions, and viral particles of the miR-138-5p mimics (lv-miR-138-m), miR-138-5p inhibitor (lv-miR-138-i) and non-specific control (lv-control) were collected and the titers were determined by fluorescence-activated cell sorting (FACSCanto II flow cytometer; BD Biosciences, San Jose, CA, USA). The pancreatic cells were subsequently transfected with lentiviruses using Lipofectamine 2000 (Invitrogen Life
Technologies, Carlsbad, CA, USA), according to manufacturer's instructions.

Reverse transcription-quantitative polymerase chain reaction $(R T-q P C R)$. The total RNA and miRNA fractions were isolated from tissues and cell lines using TRIzol reagent, according to the manufacturer's instructions (Invitrogen Life Technologies). The total RNA concentrations were measured using a NanoDrop ND-1000 spectrophotometer (NanoDrop Technologies, Wilmington, DE, USA) at 260 and $280 \mathrm{~nm}$ (A260/280) and examined with an Agilent 2100 Bioanalyzer (Agilent Technologies, Santa Clara, CA, USA). The expression of miRNA was quantified using SYBR Green. The primers for amplifying miR-138-5p were a commercial product from RiboBio. RT-qPCR was performed using the TaqMan miRNA assay (Applied Biosystems), according to the manufacturer's instructions. The amplification conditions were as follows: 35 cycles of denaturing (at $94^{\circ} \mathrm{C}$ for $60 \mathrm{sec}$ ), annealing (at $60^{\circ} \mathrm{C}$ for $60 \mathrm{sec}$ ), and chain extension (at $72^{\circ} \mathrm{C}$ for $1 \mathrm{~min}$ ), followed by a final extension step at $72^{\circ} \mathrm{C}$ for $10 \mathrm{~min}$. The expression levels of miR-138-5p and vimentin (VIM) were normalized against the expression levels of the house keeping genes, U6 and GAPDH, respectively.

MTT migration assay. For the MTT assays, the PANC-1 cells were initially plated $(10,000$ cells/well) into 96 -well plates for $24 \mathrm{~h}$ at $37^{\circ} \mathrm{C}$. The lentiviruses of lv-miR-138-m, lv-miR-138-I and lv-Control were subsequently added into the culture for 3 days, followed by replacing the culture medium with $100 \mu$ l Dulbecco's modified Eagle's medium (Invitrogen Life Technologies) $+10 \%$ FBS. MTT solution $(20 \mu 1$; Sigma-Aldrich) was added for $2 \mathrm{~h}$, according to the manufacturer's instructions. The absorbance at $490 \mathrm{~nm}$ was measured using a multifunctional plate reader (Victor 3, PerkinElmer, Turku, Finland), in order to determine the total number of viable cells.

Cell proliferation assay and 5-FU treatment. The pancreatic cancer cells were transfected with either lv-miR-138-m, lv-miR-138-i or lv-control lentivirus, and were seeded into 6 -well plates for $24 \mathrm{~h}\left(1 \times 10^{6} /\right.$ well). The viable cells were subsequently transferred at $2 \times 10^{4}$ cells/well into 24 -well plates and cultured for a further $48 \mathrm{~h}$. Cell proliferation was determined by measuring the fluorescence intensity of propidium iodide (PI), as described previously (20). At 24 and $48 \mathrm{~h}$, the fluorescence intensities were measured using a CytoFluor II multiwell plate reader (PerSeptive Biosystems, Inc., Framingham, MA, USA) and normalized against the intensity at $0 \mathrm{~h}$. All experiments were performed in triplicate. For chemo-treatment, between 1 and $100 \mu \mathrm{g} / \mathrm{ml} 5-\mathrm{FU}$ was added to the culture.

Luciferase reporter assays. Bioinformatic methods, including TargetScan (www.targetscan.org), Pictar (pictar.mdc-berlin.de) and miRANDA (www.microrna.org), were used to identify the downstream target of miR-138-5p. Regular PCR was performed on the cDNA (5 ng) of PANC-1 cells to amplify the wild-type 3'-untranslated region (UTR) and mutant 3'-UTR (modified hsa-miR-138-5p binding site) of VIM. The wild-type 3'-UTR and the mutant (mu) 3'-UTR of VIM were subsequently inserted into a luciferase reporter vector 
(pmiR-REPORT; Ambion Life Technologies, Carlsbad, CA, USA) to produce constructs of Luc-VIM and Luc-VIM-mu, and were verified by DNA sequencing. The pmiR-REPORT control vector, Luc-VIM and Luc-VIM-mu were co-transfected with $\beta$-galactosidase and Lv-miR-138-M into HEK293 cells (American Type Culture Collection) in 12-well plates using Lipofectamine 2000 (Invitrogen Life Technologies), according to the manufacturer's instructions. The luciferase activity was examined following culturing for $24 \mathrm{~h}$, using a luciferase reporter assay system (Promega Corporation, Madison, WI, USA), according to the manufacturer's instructions. The signals were normalized against the $\beta$-galactosidase activity of the control vector. The experiment was performed at least three times.

Western blotting. For western blotting, lysates of PANC-1 cells were extracted using lysis buffer (Sigma-Aldrich), containing $50 \mathrm{mM}$ Tris (pH 7.6), $150 \mathrm{mM} \mathrm{NaCl}, 1 \mathrm{mM}$ EDTA, $10 \%$ glycerol, $0.5 \%$ NP-40 and protease inhibitor cocktail (Sigma-Aldrich). The protein products were subsequently resolved on 10\% SDS-PAGE gels and transferred onto nitrocellulose membranes (Invitrogen Life Technologies). The membranes were incubated with primary antibody against VIM (1:250; SC-5565; Santa Cruz Biotechnology, Santa Cruz, CA, USA), according to the manufacturer's instructions. Following incubation, the membranes were washed with phosphate-buffered saline containing Tween and incubated with horseradish peroxidase-conjugated secondary antibodies (166-2408EDU; Bio-Rad Laboratories, Hercules, CA, USA). The western blots were visualized using an enhanced chemiluminesence system (Amersham Biosciences, Piscataway, NJ, USA), according to the manufacturer's instructions.

Transfection of siRNA. The specific VIM siRNA (VIM siRNA) and its non-specific scramble siRNA (NC_siRNA) were purchased from Santa Cruz Biotechnology Inc., and the transfection of siRNAs was performed using Lipofectamine 2000 (Invitrogen Life Technologies), according to the manufacturer's instructions. Briefly, PANC-1 cells were transfected with either VIM_siRNA $(100 \mathrm{nM})$ or NC_siRNA $(100 \mathrm{nM})$ for $48 \mathrm{~h}$, followed by examination of cell proliferation. The efficiency of siRNA on the knock down of VIM was confirmed by RT-qPCR $48 \mathrm{~h}$ following transfection.

Statistical analysis. In vivo tumor volumes were analyzed using a one-way analysis of variance. The data are expressed as the mean \pm standard error of the mean and evaluated using Student's t-test. $\mathrm{P}<0.05$ was considered to indicate a statistically significant difference. All experiments were repeated at least three times.

\section{Results}

miR-138-5p is downregulated in pancreatic cancer cell lines and tissues. The present study used RT-qPCR to examine the expression levels of miR-138-5p in the eight pancreatic cancer cell lines, non-carcinoma epithelia and HPDE cells. It was demonstrated that the expression levels of miR-138-5p were significantly lower in all the pancreatic cancer cell lines compared with the non-carcinoma cells (Fig. 1A; $\mathrm{P}<0.05$ ).

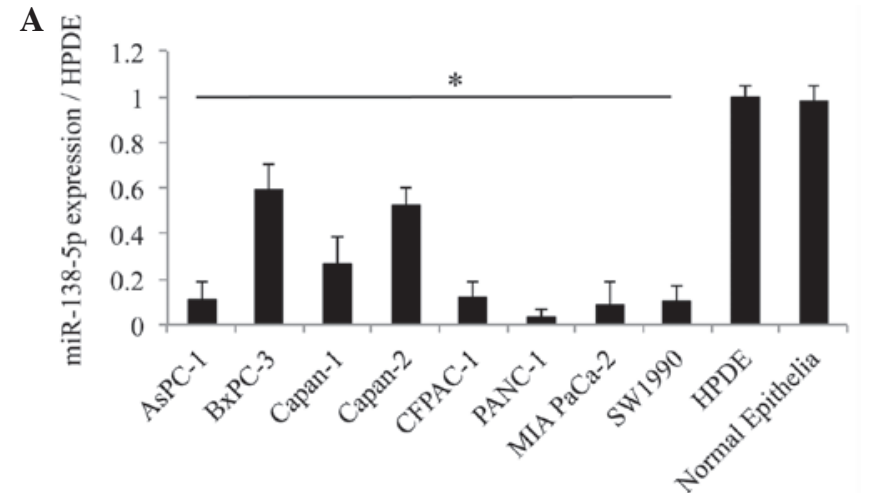

B

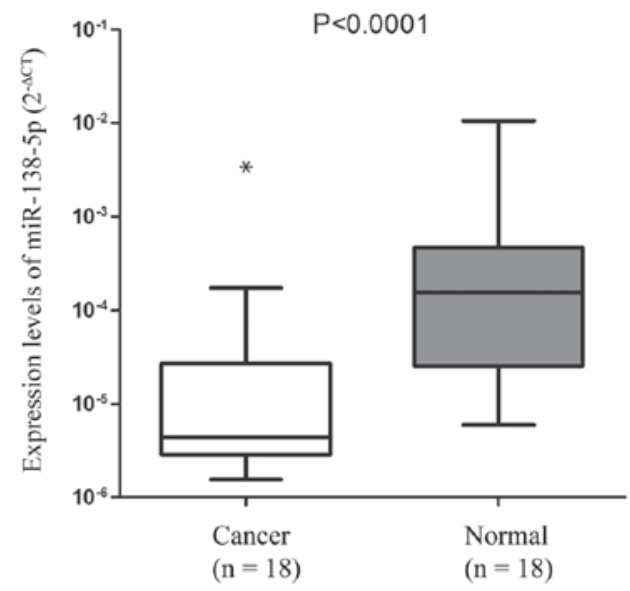

Figure 1. Expression of miR-138-5p in pancreatic cancer cell lines and primary carcinoma. (A) In eight pancreatic cancer cell lines and normal epithelia, reverse transcription-quantitative polymerase chain reaction was used to quantify the expression levels of miR-138-5p, which were normalized against the expression level in HPDE cells ( $\left.{ }^{*} \mathrm{P}<0.05\right)$. (B) In eighteen patients with pancreatic cancer, the expression levels of miR-138-5p were compared between cancer tissues and the adjacent normal tissue $\left({ }^{*} \mathrm{P}<0.05\right)$. miR, microRNA.

The expression levels of miR-138-5p in primary pancreatic carcinoma tissues and adjacent normal pancreatic epithelial tissues from 18 patients was subsequently assessed. It was demonstrated that the expression levels of miR-138-5p in cancer tissue samples was notably lower compared with the normal tissue samples (Fig. 1B; $\mathrm{P}<0.05$ ).

miR-138-5p regulates pancreatic cancer cell invasion. In order to investigate the molecular function of miR-138-5p in pancreatic cancer, PANC-1 cells were transfected with lentiviral vectors of miR-138-5p mimics (lv-miR-138-m) or miR-138-5p inhibitor (lv-miR-138-i) to genetically upregulate or downregulate the expression levels of miR-138-5p, respectively. The transfection efficiency was confirmed using RT-qPCR and demonstrated that the expression levels of miR-138-5p were significantly upregulated by lv-miR-138-m and significantly downregulated by lv-miR-138-i, compared with the expression levels of miR-138-5p in PANC-1 cells transfected with non-specific control lentivirus (lv-Control) (Fig. 2A; P<0.05).

The effect of miR-138-5p regulation on the invasive capability of PANC-1 cells was investigated. The PANC-1 cells were transfected with either lv-miR-138-m or lv-miR-138-i, to upregulate or downregulate, the endogenous expression levels 
A

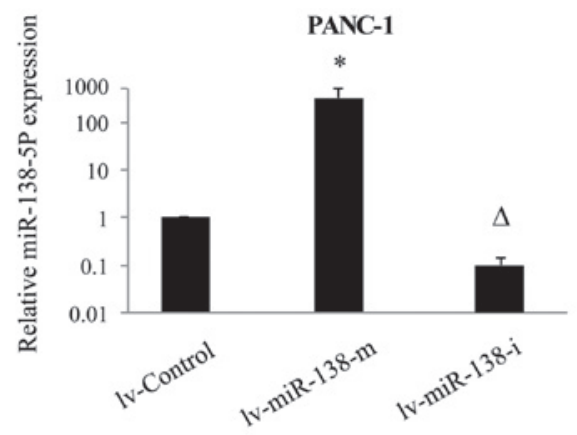

B

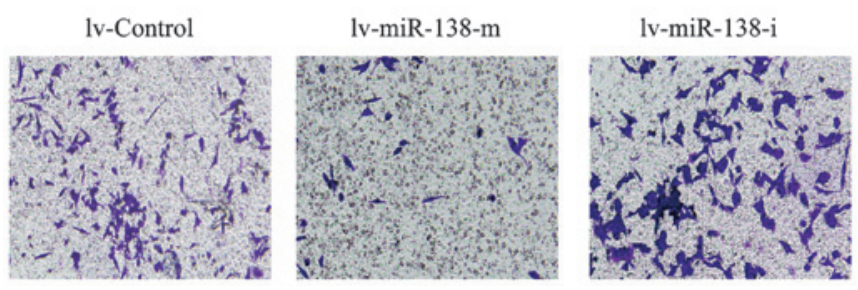

C

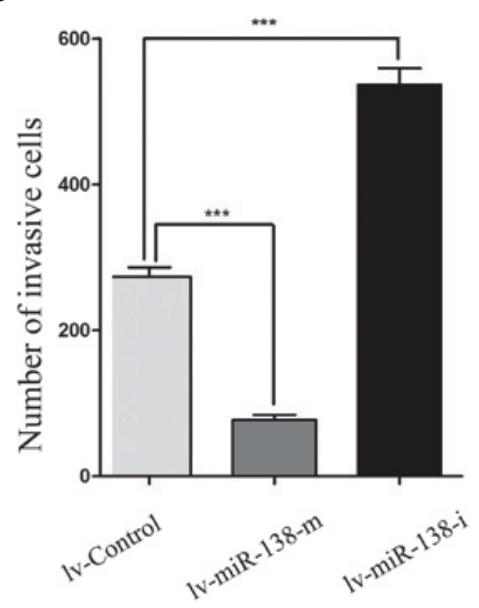

Figure 2. miR-138-5p affects pancreatic cancer cell invasion. (A) PANC-1 cells were transfected with lentiviral vector expressing miR-138-5p mimics (lv-miR-138-m; 100 pmol), miR-138-5p inhibitor (lv-miR-138-i; 100 pmol) or non-specific control vector (lv-Control; $100 \mathrm{pmol}$ ) for $24 \mathrm{~h}$. The expression levels of miR-138-5p following lentiviral transfections were measured by reverse transcription-quantitative polymerase chain reaction and normalized against the level in the lv-Control transfected PANC-1 cells $(*, \Delta \mathrm{P}<0.05)$. (B) The PANC-1 cells were transfected with lv-miR-138-m, lv-miR-138-I or lv-Control for $72 \mathrm{~h}$ and an MTT Transwell assay was performed to assess pancreatic cancer cell invasion (magnification, x100). (C) Quantification revealed that the number of invasive cells was significantly reduced by transfection with $1 \mathrm{v}-\mathrm{miR}-138-\mathrm{m}$ and were increased by transfection with lv-miR-138-I $\left.{ }^{(* * *} \mathrm{P}<0.05\right)$. miR, microRNA.

of miR-138-5p, respectively. The control cells were transfected with non-specific lv-Control. Following lentiviral transfection for 3 days, an MTT Transwell assay revealed that the upregulation of miR-138-5p significantly reduced pancreatic cancer cell invasion and downregulation significantly increased pancreatic cancer cell invasion, in the PANC-1 cells (Fig. 2B and $\mathrm{C} ; \mathrm{P}<0.05)$.

miR-138-5p regulates the chemosensitivity of pancreatic cancer cells to $5-F U$. The present study next determined whether miR-138-5p affected the chemotherapy sensitivity
A

$24 \mathrm{~h}$

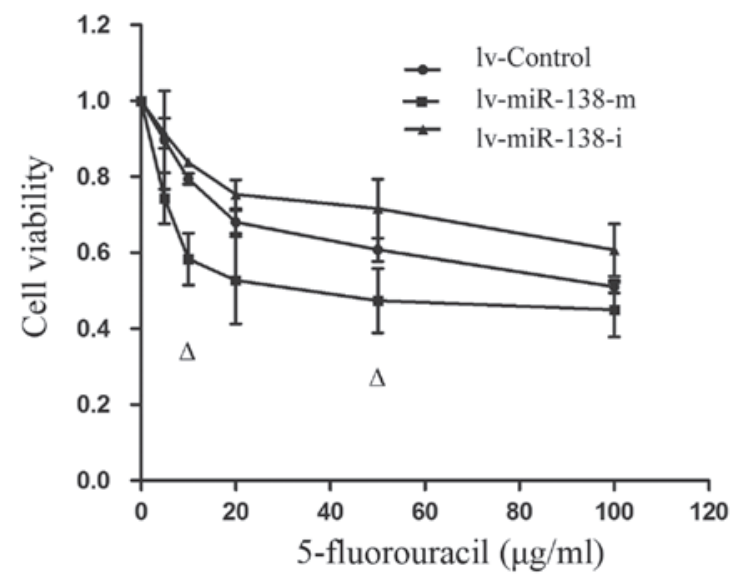

B

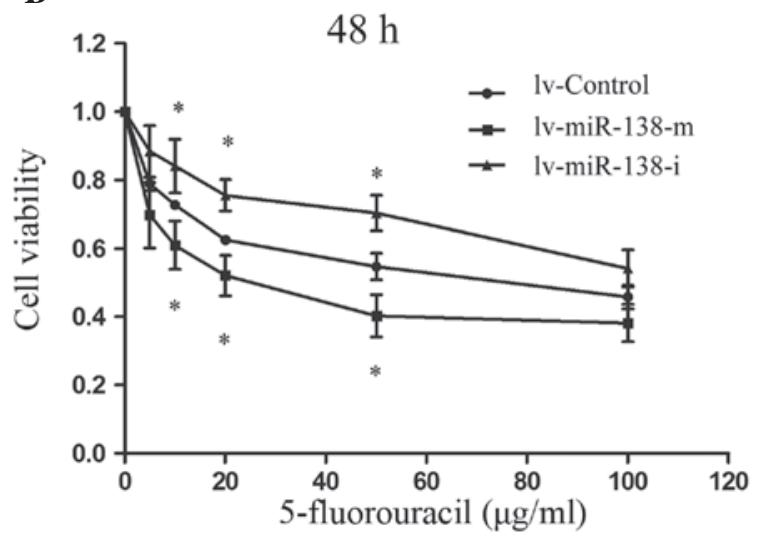

Figure 3. Effects of miR-137 on the chemosensitivity of pancreatic cancer cells to 5-fluorouracil. The PANC-1 cells were transfected with lv-miR-138-m, lv-miR-138-I or lv-Control. Their chemosensitivity was examined by the cell survival assay (A) 24 and (B) $48 \mathrm{~h}$ following treatment with 0 to $100 \mu \mathrm{g} / \mathrm{ml} 5$-fluorouracil ( ${ }^{\Delta,}{ }^{*} \mathrm{P}<0.05$, compared with the $1 \mathrm{v}$-Control). miR, microRNA.

exhibited in pancreatic cancer. PANC-1 cells were treated with various concentrations of the chemotherapeutic agent, 5-FU (between 5 and $100 \mu \mathrm{g} / \mathrm{ml}$ ), and either upregulated or downregulated expression levels of miR-138-5p. Following transfection for $24 \mathrm{~h}$, it was revealed that the upregulation of miR-138-5p significantly increased cancer cell chemosensitivity to 5 -FU at concentrations of 10 and $50 \mu \mathrm{g} / \mathrm{ml}$ (Fig. 3A; $\mathrm{P}<0.05)$. After $48 \mathrm{~h}$, the effect of miR-138-5p regulation was more clear. The upregulation of miR-138-5p significantly increased the chemosensitivity, whereas downregulation of miR-138-5p significantly decreased the chemosensitivity to 5 -FU at concentrations of 10,20 and $50 \mu \mathrm{g} / \mathrm{ml}$ (Fig. 3B; $\mathrm{P}<0.05)$.

VIM is directly regulated by miR-138-5p in pancreatic cancer. The present study next investigated the possible molecular targets associated with the regulation of miR-138-5p in pancreatic cancer. Using bioinformatic methods, including TargetScan, Pictar and miRANDA, it was demonstrated that VIM was highly likely to be the direct target of miR-138-5p (Fig. 4A). A luciferase assay was performed to confirm that miR-138-5p was directly targeting VIM in pancreatic 
A

hsa-miR-138-5p 3'...GCCGGACUAAGUGUUGUGGUCGA...5'

VIM 3'UTR WT 5',..GUGCAGCAAUAUAUUACCAGCA...3',

VIM 3' UTR MUT 5'...GUGCAGCAAUAUAUUGUGCUUA....',

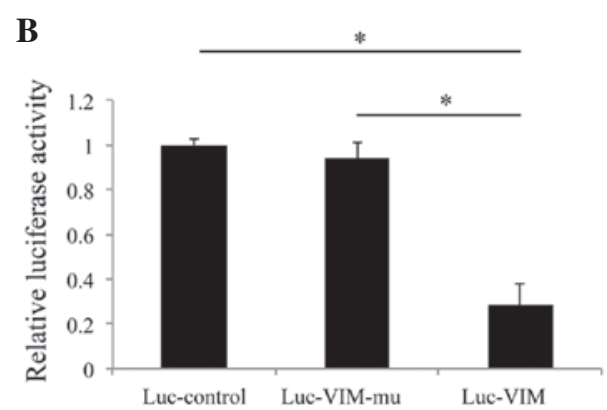

C

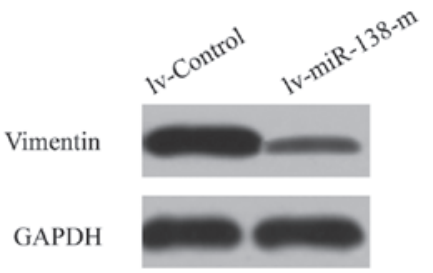

Figure 4. miR-138-5p regulates vimentin in pancreatic cancer. (A) The predicted binding site of miR-138-5p on the 3 '-UTR of VIM. The mutated 3'-UTR of VIM-mu was also listed. (B) HEK 293 T cells were transfected with $\beta$-galactosidase, lv-miR-138-5p and one of the pmiR-REPORT constructs, control construct (Luc-control), mutant 3'-UTR VIM (Luc-VIM-mu) or wild-type 3'-UTR VIM (Luc-VIM). Following culturing for $24 \mathrm{~h}$, a luciferase assay was performed. The fluorescent activities were normalized to the activity of $\beta$-galactosidase in the control vector. ("P<0.05). (C) PANC-1 cells were transfected with either lv-Control or lv-miR-138-m for $48 \mathrm{~h}$. The protein expression levels of VIM were examined by western blotting. GAPDH was used as an internal control. miR, microRNA; UTR, untranslated region; VIM, vimentin; mu, mutant.

cancer cells (Fig. 4B; P<0.05). Furthermore, western blotting revealed that lentivirus-mediated upregulation of miR-138-5p decreased the protein expression levels of VIM in PANC-1 cells, confirming that VIM is directly regulated by miR-138-5p in pancreatic cancer (Fig. 4C).

miR-138-5p-mediated pancreatic cancer invasion is also regulated by VIM. Finally, it was investigated whether the molecular target of miR-138-5p, VIM, exhibited a functional role in regulating pancreatic cancer development. siRNA was used to genetically knock down the expression levels of VIM in PANC-1 cells and cancer cell invasion was subsequently examined using an MTT migration assay. The results demonstrated that downregulation of VIM significantly reduced the invasive capability (Fig. 5A and B; NC_siRNA, vs. VIM_siRNA). These results also demonstrated that, following the downregulation of miR-138-5p in pancreatic cancer cells, knock down of VIM remained effective in reducing cancer cell invasion (Fig. 5A and B; lv-miR-138-i/NC_siRNA, vs. lv-miR-138-i/VIM_siRNA). These results suggested that VIM may act either independently, or coordinately through miR-138-5p mediation, to regulate the development of pancreatic cancer.
A
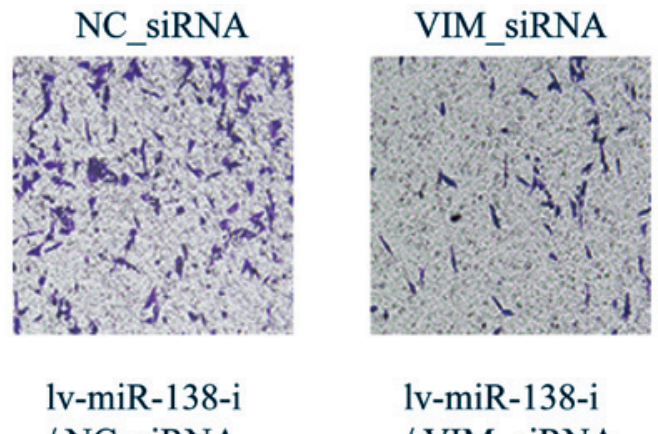

/ NC_siRNA

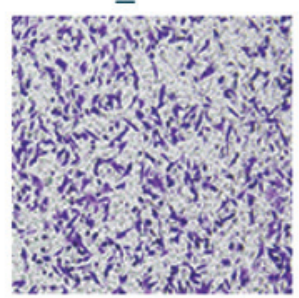

lv-miR-138-i

/ VIM_siRNA

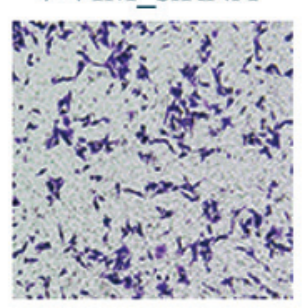

B

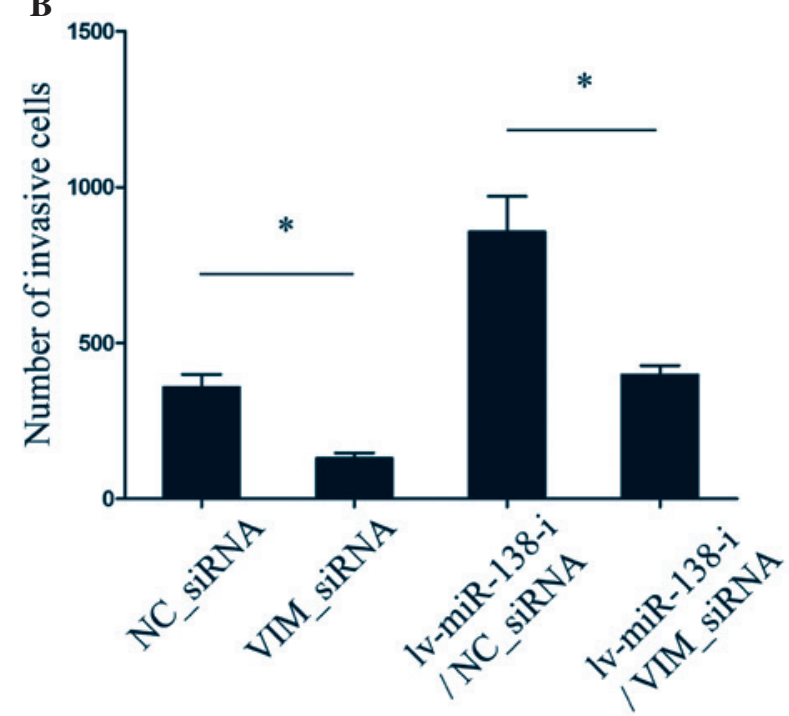

Figure 5. Knock down of VIM reduced pancreatic cancer cell invasion. (A) PANC-1 cells were transfected with either VIM siRNA (VIM_siRNA, $100 \mathrm{nM}$ ) or non-specific control siRNA (NC_siRNA, $100 \mathrm{nM}$ ), and PANC-1 cells were initially transfected with $1 \mathrm{v}-\mathrm{miR}-138$-i to downregulated the expression of miR-138-3p for $24 \mathrm{~h}$, followed by either VIM siRNA (VIM siRNA, $100 \mathrm{nM}$ ) or non-specific control siRNA (NC_siRNA, $100 \mathrm{nM}$ ) treatment. The invasion of PANC-1 cells was examined by an MTT assay (magnification, $\mathrm{x} 100)$. (B) The quantification of the numbers of invasive cells under the four experimental conditions were also quantified $\left({ }^{*} \mathrm{P}<0.05\right)$. VIM, vimentin; siRNA, small interfering RNA; miR, microRNA.

\section{Discussion}

In the present study, it was demonstrated that miR-138-5p was functionally involved in the development of pancreatic cancer cells. The results demonstrated that the expression of miR-138-5p was generally downregulated in pancreatic cancer cell lines and primary pancreatic carcinoma tissue samples. The subsequent functional assay revealed that overexpression of miR-138-5p significantly inhibited cancer cell invasion, whereas the downregulation of miR-138-5p significantly promoted invasion. These results are consistent with previous studies demonstrating that overexpressing miR-138-5p decreased cancer cell growth and increased chemosensitivity 
in other types of cancer (16-18). Therefore, it is likely that in pancreatic cancer, miR-138-5p exerts a similar functional role, acting as a tumor suppressor, as in other types of cancer, with its upregulation inhibiting and its downregulation facilitating cancer development.

The present study also demonstrated that VIM is the molecular target of miR-138-5p, by revealing that the expression level of VIM was significantly downregulated upon the overexpression of miR-138-5p in pancreatic cancer cells. It was revealed that VIM was directly involved in the regulation of pancreatic cancer development, by demonstrating that genetic knock down of VIM was also able to decrease pancreatic cancer invasion. It has been demonstrated in a previous study that VIM is commonly overexpressed in cancer tissues and is associated with cancer proliferation, migration or invasion (21). In pancreatic cancer, it was demonstrated that a high expression level of FOXC1 was notably associated with poor clinical outcomes in patients with ductal adenocarcinoma (22-25). Therefore, the results of the present study demonstrating that VIM was normally highly expressed in pancreatic cancer cell lines and cancer tissues from patients, and that downregulating VIM had an inhibitory (or anti-proliferative) effect on pancreatic cancer cell invasion, supported the hypothesis that VIM is likely a proliferative factor involved in pancreatic cancer.

In conclusion, the present study identified a novel miRNA regulator, $\mathrm{miR}-138-5 \mathrm{p}$, in pancreatic cancer and demonstrated a novel mechanism of how miR-138-5p may act as a tumor suppressor to regulate pancreatic cancer cell development. The method of upregulating miR-138-5p may provide a novel therapeutic approach for patients with pancreatic cancer.

\section{Acknowledgements}

This study was supported by the National Natural Science Foundation of China (grant no. 81160311) to JJX and the Science and Technology Foundation of Guizhou Province [grant no. Qian He J Zi (2015) 2013].

\section{References}

1. Jemal A, Siegel R, Xu J and Ward E: Cancer statistics, 2010. CA Cancer J Clin 60: 277-300, 2010.

2. Chen W, Zheng R, Zhang S, et al: Report of incidence and mortality in China cancer registries, 2009. Chin J Cancer Res 25: 10-21, 2013.

3. Siegel R, Naishadham D and Jemal A: Cancer statistics, 2013. CA Cancer J Clin 63: 11-30, 2013.

4. Hirata K, Egawa S, Kimura Y, et al: Current status of surgery for pancreatic cancer. Dig Surg 24: 137-147, 2007.
5. Gillen S, Schuster T, Meyer Zum Büschenfelde C, Friess H and Kleeff J: Preoperative/neoadjuvant therapy in pancreatic cancer: a systematic review and meta-analysis of response and resection percentages. PLoS Med 7: e1000267, 2010.

6. Pillai RS: MicroRNA function: Multiple mechanisms for a tiny RNA? RNA 11: 1753-1761, 2005.

7. Calin GA and Croce CM: MicroRNA signatures in human cancers. Nat Rev Cancer 6: 857-866, 2006.

8. Feng W and Feng Y: MicroRNAs in neural cell development and brain diseases. Sci China Life Sci 54: 1103-1112, 2011.

9. Bian S and Sun T: Functions of noncoding RNAs in neural development and neurological diseases. Mol Neurobiol 44: 359-373, 2011.

10. Papagiannakopoulos T and Kosik KS: MicroRNAs: Regulators of oncogenesis and stemness. BMC Med 6: 15, 2008.

11. Blandino G, Fazi F, Donzelli S, et al: Tumor suppressor microRNAs: A novel non-coding alliance against cancer. FEBS Lett 588: 2639-2652, 2014.

12. Palanichamy JK and Rao DS: miRNA dysregulation in cancer: Towards a mechanistic understanding. Front Genet 5: 54, 2014.

13. Wang Y, Kim S and Kim IM: Regulation of metastasis by microRNAs in ovarian cancer. Front Oncol 4: 143, 2014.

14. Othman N and Nagoor NH: The role of microRNAs in the regulation of apoptosis in lung cancer and its application in cancer treatment. Biomed Res Int 2014: 318030, 2014.

15. Han C, Yu Z, Duan Z and Kan Q: Role of microRNA-1 in human cancer and its therapeutic potentials. Biomed Res Int 2014: 428371,2014

16. Gao Y, Fan X, Li W, Ping W, Deng Y and Fu X: miR-138-5p reverses gefitinib resistance in non-small cell lung cancer cells via negatively regulating $G$ protein-coupled receptor 124 . Biochem Biophys Res Commun 446: 179-186, 2014.

17. Wang W, Zhao LJ, Tan YX, Ren H and Qi ZT: MiR-138 induces cell cycle arrest by targeting cyclin D3 in hepatocellular carcinoma. Carcinogenesis 33: 1113-1120, 2012.

18. Zhao X, Yang L, Hu J and Ruan J: miR-138 might reverse multidrug resistance of leukemia cells. Leuk Res 34: 1078-1082, 2010.

19. Radulovich N, Qian JY and Tsao MS: Human pancreatic duct epithelial cell model for KRAS transformation. Methods Enzymol 439: 1-13, 2008.

20. Zhang L, Mizumoto K, Sato N, et al: Quantitative determination of apoptotic death in cultured human pancreatic cancer cells by propidium iodide and digitonin. Cancer Lett 142: 129-137, 1999.

21. Singh S, Sadacharan S, Su S, Belldegrun A, Persad S and Singh G: Overexpression of vimentin: Role in the invasive phenotype in an androgen-independent model of prostate cancer. Cancer Res 63: 2306-2311, 2003.

22. Wang L, Gu F, Liu CY, Wang RJ, Li J and Xu JY: High level of FOXC1 expression is associated with poor prognosis in pancreatic ductal adenocarcinoma. Tumour Biol 34: 853-858, 2013.

23. Li ZM, Wen YJ, Yang HB, et al: Enhanced expression of human vimentin intermediate filaments in hepatocellular carcinoma cells decreases their proliferative and invasive abilities in vitro. Zhonghua Zhong Liu Za Zhi 30: 408-412, 2008 (In Chinese).

24. Gilles C, Polette M, Mestdagt M, et al: Transactivation of vimentin by beta-catenin in human breast cancer cells. Cancer Res 63: 2658-2664, 2003.

25. Korsching E, Packeisen J, Liedtke C, et al: The origin of vimentin expression in invasive breast cancer: Epithelial-mesenchymal transition, myoepithelial histogenesis or histogenesis from progenitor cells with bilinear differentiation potential? J Oathol 206: 451-457, 2005. 